\title{
Logarithmic structure of the generalized bifurcation set
}

\author{
by S. JANECZKO (Warszawa)
}

\begin{abstract}
Let $G: \mathbb{C}^{n} \times \mathbb{C}^{r} \rightarrow \mathbb{C}$ be a holomorphic family of functions. If $\Lambda \subset \mathbb{C}^{n} \times \mathbb{C}^{r}$, $\pi_{r}: \mathbb{C}^{n} \times \mathbb{C}^{r} \rightarrow \mathbb{C}^{r}$ is an analytic variety then

$$
Q_{\Lambda}(G)=\left\{(x, u) \in \mathbb{C}^{n} \times \mathbb{C}^{r}: G(\cdot, u) \text { has a critical point in } \Lambda \cap \pi_{r}^{-1}(u)\right\}
$$

is a natural generalization of the bifurcation variety of $G$. We investigate the local structure of $Q_{\Lambda}(G)$ for locally trivial deformations of $\Lambda_{0}=\pi_{r}^{-1}(0)$. In particular, we construct an algorithm for determining logarithmic stratifications provided $G$ is versal.
\end{abstract}

1. Introduction. Motivation of this paper lies in theoretical questions in optics where a central role is played by isotropic, Lagrangian and coisotropic varieties in a symplectic space. The geometrical framework convenient for investigations of these varieties is based mainly on the action of symplectic relations (cf. [4]).

Let $\Omega=\left(T^{*} \mathbb{R}^{k} \times T^{*} \mathbb{R}^{n}, \pi_{2}^{*} \omega_{\mathbb{R}^{n}}-\pi_{1}^{*} \omega_{\mathbb{R}^{k}}\right)$ be a product symplectic space. Lagrangian submanifolds of $\Omega$ (symplectic relations) act on subsets of $\left(T^{*} \mathbb{R}^{k}, \omega_{\mathbb{R}^{k}}\right)$ preserving their symplectic properties. In this way one can investigate the symplectic projections $\left.\pi_{\mathbb{R}^{n}}\right|_{S}: S \rightarrow \mathbb{R}^{n}$ using the representation of $S$ as the image under a symplectic relation $L \subset \Omega$ of a subset $\Lambda$ of the zero-section of $T^{*} \mathbb{R}^{k}$, i.e.

$$
S=L(\Lambda)=\left\{p \in T^{*} \mathbb{R}^{n}: \exists_{\bar{p} \in \Lambda}(\bar{p}, p) \in L\right\} .
$$

For practical purposes one seeks to classify germs of the projections $\left.\pi_{\mathbb{R}^{n}}\right|_{S}$ and describe the structure of the corresponding variety of critical values. Assuming that $L$ is generated by a smooth function $G: \mathbb{R}^{k} \times \mathbb{R}^{n} \rightarrow \mathbb{R}$ we easily find that this variety is defined as a generalized bifurcation diagram

$$
Q_{\Lambda}(G)=\left\{q \in \mathbb{R}^{n}: G(\cdot, q) \text { has a critical point belonging to } \Lambda\right\} .
$$

In this paper we study the generalized bifurcation varieties of complex analytic families $G$ using the technical tools of the theory of singularities

1991 Mathematics Subject Classification: Primary 58C27, 58F14; Secondary 57R45, $53 \mathrm{~A} 04$.

Key words and phrases: bifurcations, singularities, logarithmic stratifications. 
of functions on varieties (cf. [3]). In Section 2 we provide the classification scheme of such varieties and introduce the notion of logarithmic stratification. In Section 3 we adapt to our $\Lambda$-bifurcation varieties the method for construction of logarithmic vector fields which is well known for the standard bifurcation and discriminant varieties (cf. [2,14]). The specific algorithm explicitly calculating the tangent vector fields to $Q_{\Lambda}(G)$ and the representative examples of $\Lambda$-bifurcation varieties are discussed in Section 4 .

2. Classification of generalized bifurcation varieties. Let $\mathcal{O}_{n}$ be the ring of germs of holomorphic functions at $0 \in \mathbb{C}^{n}$. Let $(\Lambda, 0) \subset\left(\mathbb{C}^{n}, 0\right)$ be the germ of a reduced analytic subvariety of $\mathbb{C}^{n}$ at 0 :

$$
\Lambda=\left\{x \in \mathbb{C}^{n}: F(x)=0\right\}, \quad F \in \mathcal{O}_{n} .
$$

The group of germs of diffeomorphisms $\phi:\left(\mathbb{C}^{n}, 0\right) \rightarrow\left(\mathbb{C}^{n}, 0\right)$ which preserve $\Lambda$ is denoted by $\mathcal{G}_{\Lambda}$. If $J_{\Lambda}$ denotes the ideal in $\mathcal{O}_{n}$ consisting of germs of functions vanishing on $\Lambda$, then for $\phi \in \mathcal{G}_{\Lambda}$ the induced isomorphism $\phi^{*}$ : $\mathcal{O}_{n} \rightarrow \mathcal{O}_{n}$ preserves $J_{\Lambda}$.

Two function-germs $g_{1}, g_{2}:\left(\mathbb{C}^{n}, 0\right) \rightarrow(\mathbb{C}, 0)$ are called $\mathcal{G}_{\Lambda^{-}}$equivalent if there is a diffeomorphism $\phi \in \mathcal{G}_{\Lambda}$ with $g_{1} \circ \phi=g_{2}[3,10]$.

We obtain elements of $\mathcal{G}_{\Lambda}$ by integrating vector fields tangent to $\Lambda$.

Definition 2.1. We denote by $\Xi_{\Lambda}$ the $\mathcal{O}_{n}$-module of logarithmic vector fields for $\Lambda$, i.e. holomorphic vector fields on $\left(\mathbb{C}^{n}, 0\right)$, which, if considered as derivations, say $v: \mathcal{O}_{n} \rightarrow \mathcal{O}_{n}$, satisfy

$$
v . h \in J_{\Lambda} \quad \text { for all } h \in J_{\Lambda} \text {. }
$$

Modules of holomorphic vector fields of this type are discussed in [11].

A function-germ $g:\left(\mathbb{C}^{n}, 0\right) \rightarrow(\mathbb{C}, 0)$ is $k$ - $\mathcal{G}_{\Lambda^{-}}$-determined if for all $\widetilde{g}:\left(\mathbb{C}^{n}, 0\right) \rightarrow(\mathbb{C}, 0)$ with the same $k$-jet as $g$ the germs $g$ and $\widetilde{g}$ are $\mathcal{G}_{\Lambda^{-}}$equivalent. Given a germ $h:\left(\mathbb{C}^{n}, 0\right) \rightarrow(\mathbb{C}, 0)$, a germ $G:\left(\mathbb{C}^{n} \times \mathbb{C}^{r}, 0\right) \rightarrow$ $(\mathbb{C}, 0)$ is called a deformation of $h$ if $G(x, 0)=h(x)$. Formally we look on a deformation of $h$ as a pair $(G, r)$. Given two deformations $(H, r),(G, q)$ of $h$, a morphism $(\Phi, l): H \rightarrow G$ between them is defined as follows:

1. $\Phi:\left(\mathbb{C}^{n} \times \mathbb{C}^{r}, 0\right) \rightarrow\left(\mathbb{C}^{n} \times \mathbb{C}^{q}, 0\right)$ has the form $\Phi(x, u)=(\phi(x, u), u)$ with $\phi(\cdot, 0)=\operatorname{id}_{\mathbb{C}^{n}}$ and $\phi(\cdot, u) \in \mathcal{G}_{\Lambda}$ for all $u$ near $0 \in \mathbb{C}^{r}$.

2. $l:\left(\mathbb{C}^{r}, 0\right) \rightarrow\left(\mathbb{C}^{q}, 0\right)$ is such that

$$
G(\phi(x, u), l(u))=H(x, u) .
$$

A deformation $(G, q)$ of $h$ is $\mathcal{G}_{\Lambda^{-}}$versal if for any unfolding $(H, r)$ of $h$ there is a morphism $(\Phi, l): H \rightarrow G$.

Two deformations of $h$ are equivalent if there exists a morphism between them which is an isomorphism. 
Let $U \subset\left(\mathbb{C}^{n}, 0\right)$ be an open, sufficiently small subset of $\mathbb{C}^{n}$. We can also consider the sheaf $\mathcal{O}_{U}$ of holomorphic functions on $U$, and the sheaf $\operatorname{Der}_{U}$ of holomorphic vector fields on $U$, together with its subsheaf $\Xi_{\Lambda}$. Following [11] we introduce the logarithmic stratification of $U$ determined by $\Xi_{\Lambda}$.

DeFInItion 2.2. Let $\left\{\Lambda_{\alpha}: \alpha \in I\right\}$ be a stratification of $U$ with the following properties:

1. Each stratum $\Lambda_{\alpha}$ is a smooth connected immersed submanifold of $U$ and $U=\bigcup_{\alpha \in I} \Lambda_{\alpha}$.

2. If $x \in \Lambda_{\alpha}$ then $T_{x} \Lambda_{\alpha}$ coincides with $\Xi_{\Lambda}(x)$.

3. If $\Lambda_{\alpha}, \Lambda_{\beta}$ are two distinct strata with $\Lambda_{\alpha}$ meeting the closure $\bar{\Lambda}_{\beta}$ of $\Lambda_{\beta}$, then $\Lambda_{\alpha}$ is contained in the boundary $\partial \Lambda_{\beta}$ of $\Lambda_{\beta}$.

Then $\left\{\Lambda_{\alpha}: \alpha \in I\right\}$ is called a logarithmic stratification of $\Lambda$ and $\Lambda_{\alpha}$ is a logarithmic stratum.

For any variety $\Lambda$ and sufficiently small $U$ there always exists a unique logarithmic stratification of $U$.

The aim of this note is to construct the logarithmic stratification for generalized bifurcation varieties, and so to construct an appropriate module of logarithmic vector fields $\Xi_{\Lambda}$.

Let $g:\left(\mathbb{C}^{n}, 0\right) \rightarrow(\mathbb{C}, 0), g \in \mathcal{O}_{n}$. We define the Jacobi ideal of $g$ by

$$
\Delta_{\Lambda}(g)=\left\{v . g: v \in \Xi_{\Lambda}\right\} .
$$

If $\Delta_{\Lambda}(g) \supset \mathbf{m}_{n}^{k}$, then $g$ is $(k+1)-\mathcal{G}_{\Lambda}$-determined, i.e. for all $\widetilde{g}:\left(\mathbb{C}^{n}, 0\right) \rightarrow$ $(\mathbb{C}, 0)$ with the same $(k+1)$-jet as $g$ the germs $g, \widetilde{g}$ are $\mathcal{G}_{\Lambda}$-equivalent. Here $\mathbf{m}_{n}$ is the maximal ideal of $\mathcal{O}_{n}$. As in the usual singularity theory setting [1] a deformation $(G, r)$ of $g$ is $\mathcal{G}_{\Lambda^{-}}$-versal if and only if

$$
\frac{\partial G}{\partial u_{1}}(x, 0), \ldots, \frac{\partial G}{\partial u_{r}}(x, 0)
$$

$\operatorname{span} \mathcal{O}_{n} / \Delta_{\Lambda}(g)$

We know (cf. [2]) that if the set-germ

$$
\left\{x \in \mathbb{C}^{n}: v \cdot g(x)=0 \text { for all } v \in \Xi_{\Lambda}\right\}
$$

at 0 is $\{0\}$ or empty then $g$ has a $\mathcal{G}_{\Lambda}$-versal deformation. If the number $\mu=\operatorname{dim}_{\mathbb{C}} \mathcal{O}_{n} / \Delta_{\Lambda}(g)$ is finite, then it is called the multiplicity of $g$ on $\Lambda$ at 0 , and is also denoted by $\mu_{\Lambda}(g)$.

Let $(G, r)$ be a deformation of $g$.

Definition 2.3. The analytic variety

$$
Q_{\Lambda}(G)=\left\{u \in \mathbb{C}^{r}: G(\cdot, u) \text { has a critical point on } \Lambda\right\}
$$

is called the $\Lambda$-bifurcation variety of the family $G$. 
Define

$$
\Sigma_{\Lambda}(G)=\left\{(x, u) \in \mathbb{C}^{n} \times \mathbb{C}^{r}: \frac{\partial G}{\partial x_{i}}(x, u)=0, F(x)=0\right\},
$$

where $\Lambda=F^{-1}(0), F \in \mathcal{O}_{n}$. Then we see that

$$
Q_{\Lambda}(G)=\pi_{r}\left(\Sigma_{\Lambda}(G)\right)
$$

where $\pi_{r}: \mathbb{C}^{n} \times \mathbb{C}^{r} \rightarrow \mathbb{C}^{r}$.

EXAMPLE 2.4. As a natural example we consider the simplest 4 -bifurcation varieties corresponding to singularities of functions on regular boundaries (cf. [1]). Let $\Lambda=\left\{(y, x) \in \mathbb{C}^{n+1}: y=0\right\}, x=\left(x_{1}, \ldots, x_{n}\right)$. It is easy to check that for $B_{k}$ and $C_{k}$ singularities $Q_{\Lambda}(G)$ are smooth hypersurfaces. For the $F_{4}$ singularity

$$
G(y, x, u)=y^{2}+x^{3}+u_{1} x y+u_{2} y+u_{3} x
$$

the $\Lambda$-bifurcation variety $Q_{\Lambda}(G)$ is the Whitney cross cap

$$
3 u^{2}+u_{3} u_{1}^{2}=0 \text {. }
$$

By straightforward calculations we prove that for unimodal, corank one boundary singularities of smallest codimension $\mu=6$ :

$$
\begin{aligned}
& F_{1,0}: G(y, x, u)=x^{3}+b x^{2} y+y^{3}+u_{1} x y^{2}+u_{2} x y+u_{3} y^{2}+u_{4} x+u_{5} y, \\
& K_{4,2}: G(y, x, u)=x^{4}+a x^{2} y+y^{2}+u_{1} x^{2} y+u_{2} x^{2}+u_{3} y x+u_{4} x+u_{5} y,
\end{aligned}
$$

the $\Lambda$-bifurcation varieties are:

1. The trivial extension of the Whitney cross cap variety in the case $F_{1,0}$.

2. The generalized Whitney cross cap (cf. [1], Section 9.6), given in the following parametric form:

$$
u_{1}=s, \quad u_{2}=t, \quad u_{3}=w, \quad u_{4}=-4 x^{3}-2 t x, \quad u_{5}=-(a+s) x^{2}-w x .
$$

For simplest unimodal, corank two boundary singularity of type $L_{6}$ :

$$
G(y, x, u)=x_{1}^{2} x_{2}+x_{2}^{3}+y x_{1}+a y x_{2}+u_{1} y x_{2}+u_{2} x_{1}^{2}+u_{3} x_{1}+u_{4} x+u_{5} y
$$

the $\Lambda$-bifurcation variety $Q_{\Lambda}(G)$ is parametrized in the form

$u_{1}=s, u_{2}=t, u_{3}=-2 x_{1} x_{2}-2 x_{1} t, u_{4}=-x_{1}^{2}-3 x_{2}^{2}, u_{5}=-x_{1}-s x_{2}-a x_{2}$ and is an opening of the $\Sigma^{2}$-Boardmann singular mapping $\mathbb{C}^{4} \rightarrow \mathbb{C}^{4}$.

3. Logarithmic vector fields. We denote by $\operatorname{Sing}\left(\Sigma_{\Lambda}(G)\right)$ the singular part of $\Sigma_{\Lambda}(G)$. Then $\Sigma_{\Lambda}(G)-\operatorname{Sing}\left(\Sigma_{\Lambda}(G)\right)$ decomposes into analytic strata $\Sigma_{\Lambda}^{\alpha}(G), \alpha \in I$. We consider the family of mappings $\pi_{r}^{\alpha}=\left.\pi_{r}\right|_{\Sigma_{\Lambda}^{\alpha}(G)}$. Critical points of these mappings are described by an extra $n$ equations:

$$
\operatorname{rank}\left(\begin{array}{c}
\partial^{2} G / \partial x_{i} \partial x_{j} \\
\partial F / \partial x_{j}
\end{array}\right)(x, u)<n .
$$


We denote by $\Gamma_{r}^{\alpha}=\Gamma\left(\pi_{r}^{\alpha}\right)$ the set of critical values of the mapping $\pi_{r}^{\alpha}$.

Now we assume that $(G, r)$ is a $\mathcal{G}_{\Lambda}$-versal deformation of $g$. Let $g_{0}, \ldots$ $\ldots, g_{\mu-1}$ be a basis of the quotient space $\mathcal{O}_{n} / \Delta_{\Lambda}(g)$ with $g_{0}=1$ and $g_{i} \in \mathbf{m}_{n}$. Then by the equivalence of deformations we get a miniversal deformation of $g \in \mathbf{m}_{n}^{2}$ (with minimal number of deformation parameters $u$ ), i.e.

$$
G(x, u)=\sum_{i=1}^{\mu-1} u_{i} g_{i}(x)+g(x) .
$$

Now we have the following

Proposition 3.1. If $\xi \in \Xi_{Q_{\Lambda}(G)}$ then $\xi$ is $\pi_{r}$-liftable, i.e. there exists a germ of a holomorphic vector field $\widetilde{\xi}$ on $\mathbb{C}^{n} \times \mathbb{C}^{r}$ which is tangent to $\Sigma_{\Lambda}(G)$ at 0 and

$$
\xi \circ \pi_{r}=d \pi_{r} \circ \widetilde{\xi}
$$

Proof. We see that $\xi$ lifts by $\pi_{r}$ at every point $u \in \mathbb{C}^{r}$ outside $\pi_{r}\left(\operatorname{Sing}\left(\Sigma_{\Lambda}(G)\right)\right) \cup \bigcup_{\alpha \in I} \Gamma_{r}^{\alpha}$ to a holomorphic vector field $\widetilde{\xi}^{\prime}$ on $\mathbb{C}^{n} \times \mathbb{C}^{r}$ tangent to $\Sigma_{\Lambda}(G)$ and defined off a set of codimension 2 in $\mathbb{C}^{n} \times \mathbb{C}^{r}$, namely

$$
\mathbb{C}^{n} \times \pi_{r}\left(\operatorname{Sing}\left(\Sigma_{\Lambda}(G)\right)\right) \cup \bigcup_{\alpha \in I} \Gamma_{r}^{\alpha} .
$$

By Hartog's extension theorem [9], $\widetilde{\xi}^{\prime}$ extends to a holomorphic vector field $\widetilde{\xi}$ tangent to $\Sigma_{\Lambda}(G)$.

Now following the methods introduced in $[3,14]$ we give an algorithm for construction of the module $\Xi_{Q_{\Lambda}(G)}$ of vector fields for versal $G$. This algorithm is a generalization of a similar one constructed in [7] for vector fields tangent to the usual bifurcation varieties.

By Proposition 3.1, to obtain elements of $\Xi_{Q_{\Lambda}(G)}$ we have to construct all $\pi_{r}$-lowerable vector fields $\widetilde{\xi}$ tangent to $\Sigma_{\Lambda}(G)$.

Now we define the ideal

$$
J_{\Sigma_{\Lambda}(G)}=\left\langle\frac{\partial G}{\partial x_{1}}(x, u), \ldots, \frac{\partial G}{\partial x_{n}}(x, u), F(x)\right\rangle \mathcal{O}_{n+r} .
$$

Then the germ of the vector field

$$
\widetilde{\xi}=\sum_{i=1}^{n} \beta_{i} \frac{\partial}{\partial x_{i}}+\sum_{j=1}^{r} \gamma_{j} \frac{\partial}{\partial u_{j}}, \quad \beta_{i}, \gamma_{j} \in \mathcal{O}_{n+r},
$$

at $0 \in \mathbb{C}^{n} \times \mathbb{C}^{r}$, which is tangent to $\Sigma_{\Lambda}(G)$, has the property

$$
\begin{aligned}
\widetilde{\xi}\left(\frac{\partial G}{\partial x_{i}}(x, u)\right) & \in J_{\Sigma_{\Lambda}(G)}, \quad i=1, \ldots, n, \\
\widetilde{\xi}(F(x)) & \in J_{\Sigma_{\Lambda}(G)} .
\end{aligned}
$$


Lemma 3.2. Let

$$
\xi=\sum_{i=1}^{r} \alpha_{i}(u) \frac{\partial}{\partial u_{i}}, \quad \xi \in \Xi_{Q_{\Lambda}(G)} .
$$

The vector field $\widetilde{\xi} \in \Xi_{\Sigma_{\Lambda}(G)}$ is a lifting of $\xi$ if and only if for some $\beta_{i} \in \mathcal{O}_{n+r}$ and $v_{i} \in \Xi_{\Lambda}, i=1, \ldots, n$, we have

$$
\sum_{j=1}^{n} \beta_{j} v_{j}\left(\frac{\partial G}{\partial x_{i}}(x, u)\right)+\sum_{j=1}^{\mu-1} \alpha_{j}(u) \frac{\partial g_{j}}{\partial x_{i}} \in J_{\Sigma_{\Lambda}(G)},
$$

where $G$ is $\mathcal{G}_{\Lambda}$-versal,

$$
G(x, u)=\sum_{i=1}^{\mu-1} u_{i} g_{i}(x)+g(x) .
$$

Pro of. By straightforward check of the conditions (1) and (2).

Now we use the arguments working for the bifurcation and discriminant sets. Consider the ideal

$$
\widetilde{\Delta}_{\Lambda}(G)=\left\langle v_{i} \cdot G\right\rangle \mathcal{O}_{n+r}
$$

in $\mathcal{O}_{n+r}$, where $v_{i}$ are generators of $\Xi_{\Lambda}$. Since $G$ is $\mathcal{G}_{\Lambda}$-versal, by the preparation theorem the quotient module

$$
A=\mathcal{O}_{n+r} / \widetilde{\Delta}_{\Lambda}(G)
$$

is a free $\mathcal{O}_{r}$-module generated by $1, g_{1}, \ldots, g_{\mu-1}$. In fact, take $\pi(x, u) \rightarrow u$, and look on $A$ as an $\mathcal{O}_{n+r}$-module. Then $A$ is a finite $\mathcal{O}_{r}$-module if and only if $A /\left(\pi^{*} \mathbf{m}_{r}\right) A$ is finite over $\mathbb{C}$. We see that

$$
\begin{aligned}
A /\left(\pi^{*} \mathbf{m}_{r}\right) A & \cong \mathcal{O}_{n+r} /\left(\left\langle v_{i} . G\right\rangle+\mathbf{m}_{r} \mathcal{O}_{n+r}\right) \\
& \cong \mathcal{O}_{n} /\left\langle v_{i} . G(x, 0)\right\rangle \mathcal{O}_{n} \cong\left\{1, g_{1}, \ldots, g_{\mu-1}\right\}_{\mathbb{C}} .
\end{aligned}
$$

Thus for any $h \in \mathcal{O}_{n+r}$ we can write

$$
h(x, u)=\sum_{i=1}^{n} \beta_{i}(x, u)\left(v_{i} \cdot G\right)(x, u)+\sum_{j=1}^{\mu-1} \alpha_{j}(u) g_{j}(x)+\alpha(u)
$$

for some $\beta_{i} \in \mathcal{O}_{n+r}, \alpha_{i} \in \mathcal{O}_{r}$ and $\alpha \in \mathcal{O}_{r}$.

Now we have the basic result.

Theorem 3.3. Let $h \in \mathcal{O}_{n+r}$ and suppose that

Then the vector field

$$
\frac{\partial h}{\partial x_{i}}(x, u) \in J_{\Sigma_{\Lambda}(G)}, \quad i=1, \ldots, n .
$$

$$
\xi=\sum_{i=1}^{r} \alpha_{i}(u) \frac{\partial}{\partial u_{i}},
$$


where $\alpha_{i}, 1 \leq i \leq \mu-1$, are defined in (4) and $\alpha_{i}, i \geq \mu$, are arbitrary holomorphic functions from $\mathcal{O}_{r}$, is tangent to the $\Lambda$-bifurcation variety of the family $G$.

Proof. Take $h$ in the form (4). For derivatives of $h$ we have

$$
\frac{\partial h}{\partial x_{i}}(x, u)=\sum_{j=1}^{n} \frac{\partial \beta_{j}}{\partial x_{i}}\left(v_{j} \cdot G\right)+\sum_{j=1}^{n} \beta_{j} \frac{\partial}{\partial x_{i}}\left(v_{j} \cdot G\right)+\sum_{j=1}^{\mu-1} \alpha_{j}(u) \frac{\partial g_{j}}{\partial x_{i}}(x)
$$

and by assumptions this belongs to $J_{\Sigma_{\Lambda}(G)}$. We also have

$$
\sum_{j=1}^{n} \beta_{j} \frac{\partial}{\partial x_{i}}\left(v_{j} \cdot G\right)=\sum_{j=1}^{n} \beta_{j} v_{j}\left(\frac{\partial G}{\partial x_{i}}\right) \bmod \left(J_{\Sigma_{\Lambda}(G)}\right) .
$$

So by Lemma 3.2 we obtain the lifting formula (3) for the vector field $\xi=$ $\sum_{i=1}^{r} \alpha_{i} \partial / \partial u_{i}$, which is tangent to $Q_{\Lambda}(G)$.

One can also obtain the converse, which results immediately from the proof of Theorem 3.3.

COROLlaRY 3.4. Let $\xi=\sum_{i=1}^{r} \alpha_{i}(u) \partial / \partial u_{i}$ be a tangent vector field to $Q_{\Lambda}(G)$. Then for some $h \in \mathcal{O}_{n+r}$,

$$
h=\sum_{i=1}^{n} \beta_{i}\left(v_{i} G\right)+\sum_{j=1}^{\mu-1} \alpha_{j} g_{j}+\alpha,
$$

where $\beta_{i} \in \mathcal{O}_{n+r}, \alpha \in \mathcal{O}_{r}$ and $\partial h / \partial x_{i} \in J_{\Sigma_{\Lambda}(G)}$.

Proof. Take $h$ in the form (5), where

$$
\sum_{i=1}^{n} \beta_{i} v_{i}+\sum_{j=1}^{\mu-1} \alpha_{j} \frac{\partial}{\partial u_{j}} \in \Xi_{\Sigma_{\Lambda}(G)}
$$

Then by a simple check we find that $\partial h / \partial x_{i} \in J_{\Sigma_{\Lambda}(G)}$.

One can easily check that the space of germs $h \in \mathcal{O}_{n+r}$ such that $\partial h / \partial x_{i}(x, u) \in J_{\Sigma_{\Lambda}(G)}, i=1, \ldots, n$, is an $\mathcal{O}_{r}$-module, which we denote by $\mathcal{H}_{G}$.

4. An algorithm. Now we present an algorithm which is useful in obtaining all tangent vector fields to $Q_{\Lambda}(G)$. We see that

$$
\langle F\rangle J_{\Sigma_{\Lambda}(G)}+\widetilde{\Delta}_{\Lambda}^{2}(G) \subset \mathcal{H}_{G} .
$$

Since $\Delta_{\Lambda}(g)$ contains some power of the maximal ideal $\mathbf{m}_{n}$, also the space

$$
\frac{\mathcal{O}_{n}}{\Delta_{\Lambda}^{2}(g)+\langle F\rangle J_{\Lambda}(g)}, \quad J_{\Lambda}(g)=\left\langle\frac{\partial g}{\partial x_{1}}, \ldots, \frac{\partial g}{\partial x_{n}}, F(x)\right\rangle,
$$

is finite-dimensional with $\mathbb{C}$-basis, say, $\left\{f_{1}, \ldots, f_{N}\right\}$. 
By the preparation theorem $\left\{f_{i}\right\}_{i=1}^{N}$ also generates

$$
\frac{\mathcal{O}_{n+r}}{\widetilde{\Delta}_{\Lambda}^{2}(G)+\langle F\rangle J_{\Sigma_{\Lambda}(G)}}
$$

as an $\mathcal{O}_{r}$-module.

Now any element $h \in \mathcal{H}_{G}$ can be written in the form

$$
\begin{aligned}
h(x, u)= & \sum_{i=1}^{N} \phi_{i}(u) f_{i}(x)+\sum_{i, j=1}^{n} \beta_{i, j}(x, u) \frac{\partial G}{\partial x_{i}}(x, u) \frac{\partial G}{\partial x_{j}}(x, u) \\
& +\sum_{i=1}^{n} \gamma_{i}(x, u) \frac{\partial G}{\partial x_{i}}(x, u) F(x)+\gamma_{0}(x, u) F(x)^{2},
\end{aligned}
$$

where $\beta_{i, j}, \gamma_{i}, \gamma_{0} \in \mathcal{O}_{n+r}$ and we seek elements $\phi_{i} \in \mathcal{O}_{r}$ such that

$$
\sum_{i=1}^{N} \phi_{i}(u) \frac{\partial f_{i}}{\partial x_{j}} \in J_{\Sigma_{\Lambda}(G)}, \quad 1 \leq j \leq n .
$$

We show how to work with this approach and algorithm in several concrete cases.

4.1. Let $\Lambda=\left\{(y, x) \in \mathbb{C}^{n+1}: y=0\right\}, x=\left(x_{1}, \ldots, x_{n}\right)$. Then for some $g \in \mathcal{O}_{n+1}$ and the versal unfolding $G$ of $g$ we have

$$
\begin{aligned}
\Delta_{\Lambda}(g) & =\left\langle y \frac{\partial g}{\partial y}, \frac{\partial g}{\partial x_{1}}, \ldots, \frac{\partial g}{\partial x_{n}}\right\rangle \mathcal{O}_{n+1} \\
\widetilde{\Delta}_{\Lambda}(G) & =\left\langle y \frac{\partial G}{\partial y}, \frac{\partial G}{\partial x_{1}}, \ldots, \frac{\partial G}{\partial x_{n}}\right\rangle \mathcal{O}_{n+1+r} \\
J_{\Sigma_{\Lambda}(G)} & =\left\langle\frac{\partial G}{\partial y}, \frac{\partial G}{\partial x_{1}}, \ldots, \frac{\partial G}{\partial x_{n}}, y\right\rangle \mathcal{O}_{n+1+r}
\end{aligned}
$$

As an example we take the simplest nontrivial case of type $F_{4}$ (cf. [7]):

$$
g(y, x)=y^{2}+x^{3} .
$$

Then $G(y, x, u)=y^{2}+x^{3}+u_{1} x y+u_{2} y+u_{3} x$ and

$$
\begin{aligned}
& \widetilde{\Delta}_{\Lambda}^{2}(G)=\left\langle 2 y^{2}+y u_{2}+u_{1} x y, 3 x^{2}+u_{1} y+u_{3}\right\rangle, \\
& J_{\Sigma_{\Lambda}(G)}=\left\langle u_{2}+u_{1} x, 3 x^{2}+u_{3}, y\right\rangle,
\end{aligned}
$$

and also the quotient space

$$
\frac{\mathcal{O}_{2+3}}{\widetilde{\Delta}_{\Lambda}^{2}(G)+\langle y\rangle J_{\Sigma_{\Lambda}(G)}}
$$

is generated by $\left\{1, x, y, x^{2}, x^{3}, x y\right\}$ as an $\mathcal{O}_{3}$-module.

We see that the functions

$$
h(y, x, u)=\alpha_{1}(u)+\alpha_{2}(u) x+\alpha_{3}(u) x^{2}+\alpha_{4}(u) x^{3}+\alpha_{5}(u) y+\alpha_{6} x y+\psi(y, x, u)
$$


with

$$
\begin{aligned}
& \alpha_{5}(u)+\alpha_{6}(u) x \in J_{\Sigma_{\Lambda}(G)}, \\
& \alpha_{2}(u)+2 \alpha_{3}(u) x+3 \alpha_{4}(u) x^{2} \in J_{\Sigma_{\Lambda}(G)}, \\
& \psi \in \widetilde{\Delta}_{\Lambda}^{2}(G)+\langle y\rangle J_{\Sigma_{\Lambda}(G)}
\end{aligned}
$$

form the space $\mathcal{H}_{G}$.

Now it is easy to calculate the basis of vector fields tangent to $Q_{\Lambda}(G)$ (cf. [7]):

$$
\begin{aligned}
V_{1} & =-u_{1}^{2} \frac{\partial}{\partial u_{2}}+6 u_{2} \frac{\partial}{\partial u_{3}}, \\
V_{2} & =u_{1} \frac{\partial}{\partial u_{1}}+u_{2} \frac{\partial}{\partial u_{2}}, \\
V_{3} & =-u_{1} \frac{\partial}{\partial u_{1}}+2 u_{3} \frac{\partial}{\partial u_{3}}, \\
V_{4} & =3 u_{2} \frac{\partial}{\partial u_{1}}-u_{1} u_{3} \frac{\partial}{\partial u_{2}},
\end{aligned}
$$

which satisfy the relation $-u_{1} V_{4}+u_{3} V_{1}-3 u_{2} V_{3}=0$.

4.2. In the case of $\Lambda$ singular our algorithm leads to quite complicated calculations. We show only some steps of the procedure which make clear the differences with the nonsingular case.

Let $\Lambda=\left\{(x, y) \in \mathbb{C}^{2}: F(x, y)=x^{3}-y^{2}=0\right\}$. The module $\Xi_{\Lambda}$ of vector fields tangent to $\Lambda$ is generated by

$$
\xi_{1}=2 x \frac{\partial}{\partial x}+3 y \frac{\partial}{\partial y}, \quad \xi_{2}=3 x^{2} \frac{\partial}{\partial y}+2 y \frac{\partial}{\partial x} .
$$

We consider the simplest non-Morse function $g(x, y)=x^{3}+y^{2}$. Its Jacobi ideal is

$$
\Delta_{\Lambda}(g)=\left\langle x^{2} y, x^{3}+y^{2}\right\rangle
$$

and a versal deformation is

$$
G(x, y, u)=x^{3}+y^{2}+u_{1} x y^{2}+u_{2} x y+u_{3} x^{2}+u_{4} y^{2}+u_{5} x+u_{6} y .
$$

The corresponding $\Lambda$-bifurcation variety $Q_{\Lambda}(G)$ is described by the equations

$$
\begin{aligned}
& 3 x^{2}+u_{1} y^{2}+u_{2} y+2 u_{3} x+u_{5}=0, \\
& 2 y+2 u_{1} x y+u_{2} x+2 u_{4} y+u_{6}=0
\end{aligned}
$$

together with $x^{3}-y^{2}=0$.

The quotient space

$$
\frac{\mathcal{O}_{2+6}}{\widetilde{\Delta}_{\Lambda}(G)+\left\langle x^{3}-y^{2}\right\rangle J_{\Sigma_{\Lambda}(G)}}
$$


is generated by $\left\{1, x, y, x^{2}, x y, y^{2}, x^{3}, x^{2} y, x y^{2}, y^{3}, x^{2} y^{2}, x y^{3}, y^{4}\right\}$ as an $\mathcal{O}_{6^{-}}$ module. The functions

$$
\begin{aligned}
h(x, y, u)= & \alpha_{0}(u)+\alpha_{1}(u) x+\alpha_{2}(u) y+\alpha_{3}(u) x^{2}+\alpha_{4}(u) x y+\alpha_{5}(u) y^{2} \\
& +\alpha_{6}(u) x^{3}+\alpha_{7}(u) x^{2} y+\alpha_{8}(u) x y^{2}+\alpha_{9}(u) y^{3}+\alpha_{10}(u) x^{2} y^{2} \\
& +\alpha_{11}(u) x y^{3}+\alpha_{12}(u) y^{4}+\psi(x, y, u)
\end{aligned}
$$

with

$$
\begin{gathered}
\alpha_{1}(u)+2 \alpha_{3}(u) x+\alpha_{4}(u) y+3 \alpha_{6}(u) x^{2}+2 \alpha_{7}(u) x y \\
+\alpha_{8}(u) y^{2}+2 \alpha_{10}(u) x y^{2}++\alpha_{11}(u) y^{3} \in J_{\Sigma_{\Lambda}(G)}, \\
\alpha_{2}(u)+\alpha_{4}(u) x+2 \alpha_{5}(u) y+\alpha_{7}(u) x^{2}+2 \alpha_{8}(u) x y \\
+3 \alpha_{9}(u) y^{2}+2 \alpha_{10}(u) x^{2} y+3 \alpha_{11}(u) x y^{2}+4 \alpha_{12}(u) y^{3} \in J_{\Sigma_{\Lambda}(G)}, \\
\psi \in \widetilde{\Delta}_{\Lambda}^{2}(G)+\left\langle x^{3}-y^{2}\right\rangle J_{\Sigma_{\Lambda}(G)}
\end{gathered}
$$

form the space $\mathcal{H}_{G}$.

Remarks. 1. If $g$ is a Morse singularity on $\Lambda$ singular then the $\Lambda$-bifurcation variety $Q_{\Lambda}(G)$ is diffeomorphic to the product $\Lambda \times \mathbb{C}^{k}$ for some $k \in \mathbb{N} \cup\{0\}$.

2. Let $G(x, u)$ be a germ of a holomorphic family of functions. Let $\Lambda_{0} \subset \mathbb{C}^{n}$ be a germ of a complex space. We consider a deformation of $\Lambda_{0}$, i.e. a family of varieties $\pi: \widetilde{\Lambda} \rightarrow \mathbb{C}^{r}$ with $\pi^{-1}(0)=\Lambda_{0}$. As a natural generalization of a $\Lambda$-bifurcation variety of $G$ we have the $\widetilde{\Lambda}$-bifurcation variety of $G$ defined by

$Q_{\widetilde{\Lambda}}(G)=\left\{(x, u) \in \mathbb{C}^{n} \times \mathbb{C}^{r}: \frac{\partial G}{\partial x_{i}}(x, u)=0,(x, u) \in \pi^{-1}(u), i=1, \ldots, n\right\}$.

If $\widetilde{\Lambda}$ is the versal deformation of $\Lambda_{0}$ (cf. $\left.[8,5]\right)$ we may use the normal forms of $\widetilde{\Lambda}$ to consider the parametrized groups (deformations of groups) $u \rightarrow$ $\mathcal{G}_{\widetilde{\Lambda}_{u}}, \widetilde{\Lambda}_{u}=\pi^{-1}(u)$ acting on families $G$. In case of families of hypersurfaces, $\widetilde{\Lambda}$ is given by the holomorphic function $F:\left(\mathbb{C}^{n} \times \mathbb{C}^{r}, 0\right) \rightarrow(\mathbb{C}, 0), \Lambda_{u}=\{x \in$ $\left.\mathbb{C}^{n}: F(\cdot, u)=0\right\}$. So the classification problem of $\widetilde{\Lambda}$-varieties is reduced to the classification of map-germs $(F, G):\left(\mathbb{C}^{n} \times \mathbb{C}^{r}, 0\right) \rightarrow \mathbb{C}^{2}$ with right and modified left equivalences (cf. [13]).

\section{References}

[1] V. I. Arnold, S. M. Gusein-Zade and A. N. Varchenko, Singularities of Differentiable Maps, Vol. 1, Birkhäuser, Boston, 1985.

[2] J. W. Bruce, Functions on discriminants, J. London Math. Soc. (2) 30 (1984), $551-567$. 
[3] J. W. Bruce and R. M. Roberts, Critical points of functions on analytic varieties, Topology 27 (1988), 57-90.

[4] V. Guillemin and S. Sternberg, Symplectic Techniques in Physics, Cambridge Univ. Press, Cambridge, 1984.

[5] S. Izumiya, Generic bifurcations of varieties, Manuscripta Math. 46 (1984), 137-164.

[6] S. Janeczko, On isotropic submanifolds and evolution of quasicaustics, Pacific J. of Math. 158 (1993), 317-334.

[7] -, On quasicaustics and their logarithmic vector fields, Bull. Austral. Math. Soc. 43 (1991), 365-376.

[8] A. Kas and M. Schlessinger, On the versal deformation of a complex space with an isolated singularity, Math. Ann. 196 (1972), 23-29.

[9] S. Łojasiewicz, Introduction to Complex Analytic Geometry, Birkhäuser, 1991.

[10] O. W. Lyashko, Classification of critical points of functions on a manifold with singular boundary, Funktsional. Anal. i Prilozhen. 17 (3) (1983), 28-36 (in Russian).

[11] K. Saito, Theory of logarithmic differential forms and logarithmic vector fields, J. Fac. Sci. Univ. Tokyo Sect. IA Math. 27 (1980), 265-291.

[12] H. Terao, The bifurcation set and logarithmic vector fields, Math. Ann. 263 (1983), 313-321.

[13] C. T. Wall, A splitting theorem for maps into $\mathbb{R}^{2}$, ibid. 259 (1982), 443-453.

[14] V. M. Zakalyukin, Bifurcations of wavefronts depending on one parameter, Functional Anal. Appl. 10 (1976), 139-140.

INSTITUTE OF MATHEMATICS

WARSAW UNIVERSITY OF TECHNOLOGY

PL. POLITECHNIKI 1

00-661 WARSZAWA, POLAND 